\title{
RÚBRICAS COMO MÉTODO EFECTIVO DE VALORACIÓN EN LA EVALUACIÓN DEL APRENDIZAJE
}

\author{
RUBRICS AS EFFECTIVE METHOD IN THE EVALUATION \\ OF LEARNING ASSESSMENT
} en temas de evaluación del aprendizaje, y sus vínculos con las rúbricas. Se describen las características de las rúbricas como herramientas auxiliares para lograr un control pormenorizado de los objetivos de aprendizaje, así como para disponer de herramientas de rápida retroalimentación del docente a los estudiantes.

The use of rubrics is one of the contemporary use of technology for learning assessment solutions. This paper addresses the relationship between definitions, key concepts and issues relevant authors learning assessment, and its links with the rubrics. The characteristics of the rubrics as auxiliary tools are described to achieve a detailed record of the learning objectives and tools to provide quick feedback from teachers to students.

(n) 
INTRODUCCIÓN

Dado que el Consejo de Educación Superior (CES) ha promulgado una ley en la que cada alumno para obtener su título al final de su carrera será evaluado de manera individual, para las universidades del Ecuador es imprescindible contar con un sistema de evaluación continua que funcione como un sistema de aseguramiento de graduación, que se ejecute a lo largo de la vida estudiantil y que permita a ambas partes, universidad y alumno, saber en forma rápida y oportuna cuáles son los puntos en los que deben intervenir; las universidades por su parte mejorando sus competencias, y los estudiantes conociendo a ciencia cierta lo que están haciendo bien y qué están haciendo mal o insuficiente.

La estandarización de la evaluación de conocimientos, habilidades y competencias puede sustentarse en diferentes enfoques. Los autores de este trabajo consideraron como presupuestos teóricos fundamentales: enfoques de evaluación del aprendizaje; evaluación del aprendizaje y rúbricas; y las rúbricas como insumos para las tutorías virtuales.

\section{ENFOQUES DE EVALUACIÓN DEL APRENDIZAJE}

La evaluación es una práctica muy antigua, que data de hace más de tres mil años en la China Imperial como procedimiento para la selección de funcionarios (Dubois, 1970) (Coffman, 1971). También Sunberg (1977) cita pasajes bíblicos evaluadores, y Blanco (1994) se refiere a exámenes de los profesores griegos y romanos. Sin embargo, según McReynold el tratado más importante de evaluación de la antigüedad es el Tetrabiblos (atribuido a Ptolomeo) (Escudero, 2003). En tanto a la práctica pedagógica, la evaluación es sumamente utilizada en las instituciones educativas, muchas veces sin tener claro los objetivos principales que persigue, la función que cumple, los beneficiarios, y al servicio de quién se pone.

La evaluación es una tarea que todas las personas, de una forma u otra, realizan en su vida cotidiana y sobre distintos ámbitos. Con frecuencia se registran valoraciones de lo que se ha logrado con relación a una meta, es decir se evalúan los resultados.

Cuando se trabaja con un grupo de personas en proceso de formación o en situación de aprendizaje, los educadores se plantean numerosas interrogantes: ¿están aprendiendo?, ¿se están coordinando bien las actividades de aprendizaje?, ¿se eligieron convenientemente las estrategias para que los alumnos tomen contacto con los contenidos?, ¿estarán apareciendo dificultades en ellos que no se evidencian? Entonces, cuando se habla de evaluación del aprendizaje ¿de qué se está hablando?

La Real Academia de la Lengua Española apunta que evaluar es señalar el valor de una cosa. Implica además, el reconocimiento del poder. Según Gvirtz y Palamidessi (2006), "evaluar es una acción que supone el ejercicio de un poder, el poder del evaluador. El que evalúa es reconocido como una autoridad capaz de preguntar, inspeccionar, examinar, valorar, calificar, jerarquizar personas y cosas. Evaluar se parece mucho a la situación de administrar justicia. Se trata de sopesar y valorar pruebas, calificar a un individuo y tomar una decisión respecto de su situación. Evaluar es establecer un juicio acerca de una persona o cosa. El evaluador no es un mero analizador de datos sino alguien que juzga, que toma una decisión. No por casualidad la evaluación escolar se relaciona muy a menudo con el poder de premiar, castigar o vigilar» (p. 7).

El concepto de evaluación en el campo de la educación ha ido variando a lo largo del tiempo. Posteriormente, fue extendiéndose a otros aspectos educativos, hasta llegar al intento de cariz totalizador que tiene hoy en día. En este sentido, los aspectos que se evalúan remiten a diferentes procesos didácticos: el aprendizaje de los alumnos, las estrategias del docente, sus cualidades para la enseñanza y orientación, la selección de contenidos, el clima de la clase, el espacio físico del aula, los procesos desarrollados durante la enseñanza y el aprendizaje, los logros obtenidos, la valoración entre lo hecho y lo que queda por hacer, el ajuste entre la planificación del docente y el de la institución, entre muchos otros.

La evaluación toma un sentido totalizador a partir de la década del 70 del siglo XX; entendiendo por totalizador la inclusión de criterios que contemplen el funcionamiento global de un proyecto y las circunstancias en las que se desarrolle y concrete. No se evalúa únicamente el progreso y los resultados puntuales del aprendizaje de los alumnos, sino también el progreso de todo el proceso educativo.

Esta concepción de la evaluación podría asumirse como el proceso de diseño, recogida y análisis sistemático de cualquier información, para determinar el valor de lo que se hace. Es decir que la evaluación estará referida a la valoración de un proceso realizado durante un tiempo determinado, mediante procedimien- 
tos formales o informales, a medida que se va conociendo la realidad socioeducativa en la que se actúa, las posibilidades como docentes y las posibilidades de los alumnos, las tareas, los materiales curriculares utilizados y los conocimientos previos de los alumnos.

Ezequiel Ander-Egg (1993) plantea lo que la evaluación debe ser y no debe ser, a saber:

\section{Debe ser}

- Parte del proceso de enseñanza y aprendizaje.

- De carácter formativo: ayuda al proceso de aprendizaje y tiene valor pedagógico.

- Continua: no debe limitarse al momento del examen.

- Integral: comprende e integra lo conceptual, lo procedimental y lo actitudinal.

- Sistemática: se realiza de acuerdo con un plan y criterios preestablecidos.

\section{No debe ser}

- Un fin en sí misma.

- Un sistema para clasificar, seleccionar y excluir a los alumnos, de acuerdo con el mayor o menor grado de conocimiento que tengan acerca de los contenidos.

- Un proceso competitivo en el que se comparan las diferentes capacidades de los alumnos.

- Una forma de control de aprendizajes memorísticos como modo de verificar la adquisición del conocimiento.

- Una forma de averiguar lo que el alumno no sabe.

Según Stenhouse (1984) para evaluar es necesario comprender: el proceso de aprendizaje; las limitaciones y potencialidades del alumno y del docente; que la evaluación está compuesta por diversos elementos entremezclados e incidentes unos a otros, y que conocer las dificultades es un modo de superarlas.

Por otra parte, la misma complejidad de la evaluación del aprendizaje hace que ésta se convierta en un problema inquietante para unos, en un reto y desafío para algunos, en algo natural y práctico para otros, y en preocupación permanente para las instituciones que señalan los resultados de la evaluación del aprendizaje como los determinantes de la deserción escolar, la repitencia, la violencia escolar, la calidad de la educación impartida.

Sin embargo, más allá de los matices valorativos, la evaluación debe servir para crear un clima favorable al mejoramiento de los procesos del quehacer académico, debe servir de retroalimentación del proceso educativo, entonces la evaluación se entenderá como un análisis sistemático de los eventos, que ocurre como consecuencia de un programa realizado para ayudar a mejorar programas (Osorio, Muñoz, Álvarez y Arévalo, 2005), los autores de este trabajo comparten este posicionamiento en tanto se trata en lo fundamental, de mejorar el aprendizaje.

Resulta interesante considerar también las funciones que Miguel Santos (1999) le atribuye al ejercicio de evaluación del aprendizaje, a saber:

- Función diagnóstica: porque permite saber cuál es el estado cognitivo y actitudinal de los alumnos, para así ajustar la acción docente a las características de los estudiantes. Este diagnóstico es una radiografía que facilita el aprendizaje significativo y relevante de los alumnos, ya que el docente partirá de los saberes previos y además podrá considerar las actitudes y expectativas.

- Función selectiva: la evaluación permite al sistema educativo realizar la selección de los estudiantes. A través de las calificaciones, la entidad educativa clasifica a los alumnos; algunos son eliminados porque no llegan al nivel mínimo requerido, otros se van situando en otros niveles según la calificación que obtienen.

- Función jerarquizadora: la capacidad de decidir qué se debe evaluar, cómo, y cuál es el nivel mínimo requerido para aprobar, confiere un poder especial al docente. Este es un poder legal, pero no moral. En más de una oportunidad, en vez de operar como una instancia más de aprendizaje, la evaluación hace las veces de mecanismo de control.

- Función comunicadora: a través de la evaluación, el docente también se relaciona con el alumno. Este tipo de comunicación particular tiene repercusiones psicológicas, tanto para el docente como para el alumno. El estudiante ve potenciada o disminuida su autoestima, según los resultados que va obteniendo en sus evaluaciones. Se compara con sus compañeros y saca sus conclusiones, por su parte, el maestro se autovalora más de acuerdo con los resultados de los alumnos. El docente también se 
compara con otros colegas, y con dichos resultados obtenidos por sus alumnos.

- Función formativa: la evaluación puede estar al servicio de la compresión y de la formación. También permite conocer cómo se ha realizado el aprendizaje (Stenhouse, 1984), de lo que puede derivarse una toma de decisiones racional y beneficiosa para un nuevo proceso. La evaluación del aprendizaje, en su función formativa, se realiza durante el proceso, $y$ permite la retroalimentación de la práctica docente. No atiende solo los resultados obtenidos por los alumnos.

En los procesos de evaluación del aprendizaje confluyen contradicciones irreconciliables que conviven en el proceso de escolarización. Estas están determinadas por la forma de entender la evaluación: sumativa y formativa.

- La primera utiliza mecanismos tradicionales, y se caracteriza por concebir a la evaluación como la medición de productos de aprendizaje para calificar a los alumnos. Aquí la función central es la calificación del alumno; al alumno se lo califica con una nota y de esta forma se acreditan los supuestos saberes que el estudiante tiene o no tiene, además el docente no comparte la responsabilidad ante el posible fracaso del estudiante, no se analizan los errores de la evaluación, ni tampoco la pertinencia de las estrategias de aprendizaje utilizadas. Aquí los instrumentos son las típicas pruebas escritas, con ejercicios, por ejemplo, de selección múltiple cuyo resultado se plasma en el típico boletín de calificaciones. Los estándares a cumplir son estipulados por el maestro. En esta forma tradicional de evaluar el alumno no tiene ningún interés por aprender, por conocer, sino que su interés real está en aprobar (Litwin, 2004).

- La segunda, se refiere más una instancia de reflexión y análisis de la enseñanza, se plantean otros mecanismos más creativos de instrumentos de evaluación para poder emitir no solo una calificación sino un juicio complejo de valor, cuya finalidad es la comprobación de un saber. Pero además dicha valoración sirve para sopesar las estrategias de enseñanza y poder mejorarlas. Esta evaluación está orientada a recolectar datos del proceso de enseñanza y aprendizaje para mejorarlos. Se podría decir que es una evaluación que mira hacia el futuro, se preocupa por mejorar lo que queda por hacer o enseñar y se caracteriza por ser continua.

La definición más abarcadora y a la vez detallada la ofrece Miriam González (2002) cuando considera a la evaluación del aprendizaje como un caso particular de la evaluación, y la define en términos genéricos, como la actividad cuyo objetivo es la valoración del proceso y los resultados del aprendizaje de los estudiantes, a los efectos fundamentales de orientar y regular la enseñanza y contribuir al logro de las finalidades de la formación.

La opinión de los autores de este trabajo es que la evaluación del aprendizaje es, fundamentalmente, un proceso, un todo integrador, y no hitos aislados. Debe ser entendida como una categoría de la didáctica de naturaleza formativa.

\section{LA EVALUACIÓN DEL APRENDIZAJE Y LAS RÚBRICAS}

Cuando se habla de evaluación del aprendizaje, en sentido general, se refiere a un proceso a través del cual se recoge, analiza y emiten juicios de valor sobre el aprendizaje logrado por los aprendices o estudiantes, con el propósito de tomar decisiones conducentes a la mejora de la calidad de ese aprendizaje.

Evaluar es también, una capacidad que consiste en valorar la interrelación entre el producto, el objeto y el proceso. Como capacidad implica el desarrollo de ciertas destrezas concretas como diagnosticar, verificar y criticar. Diagnosticar a su vez, supone poner en juego habilidades tales como examinar, apreciar, valorar, estimar, juzgar, etc. (Casas, 2007).

Aún cuando la evaluación del aprendizaje constituye el lado débil del currículo, solo evidenciada por un corto tiempo en los procesos de formación, es necesario recalcar su gran importancia y la profunda unidad que debe guardar con la planificación curricular y los procesos de enseñanza y aprendizaje. Se evalúa lo que se planifica y lo que se aprende y enseña. Por tanto, desde la planificación se debe tener claridad sobre lo que se quiere que aprendan los estudiantes. Si las capacidades que se planifican desarrollar se limitan a la reproducción de conocimientos y/o los conceptos y no se apunta a desarrollar capacidades superiores como la movilización o resolución de problemas, entonces, 
la evaluación se verá limitada a una esfera de manejo inferior. La evaluación del aprendizaje está al servicio de la programación y ejecución curricular, pero sobre todo, al servicio del desarrollo integral de los estudiantes, de sus capacidades y valores.

La cuestión del por qué los estudiantes son evaluados podría, en principio, parecer un asunto de simple sentido común, resulta claro que su actuación necesita ser valorada. No obstante, la evaluación del alumnado ha seguido tradiciones disciplinarias o requerimientos regulatorios donde los profesores no han tenido la necesidad o el tiempo para preguntarse mucho acerca de por qué era adoptado un enfoque particular en un momento determinado. Pero las estrategias y tareas de evaluación suelen variar considerablemente entre diferentes establecimientos disciplinarios.

Al discutir lo racional de una estrategia de evaluación sobre otra, algunos profesores bien podrían tener propósitos diferentes, mientras que otros podrían esforzarse por resolver cuestiones de si un enfoque probablemente está siendo más exitoso que otro.

Para ser capaces de tomar decisiones informadas acerca de cómo evaluar a los estudiantes, los profesores tienen que tomar en cuenta los roles y propósitos de la evaluación del aprendizaje del estudiante, porque éstos podrían ser altamente diferenciados y ellos forman las concepciones de lo que es, o no, importante. Cuando se considera que la evaluación del estudiante es de interés para un rango de diferentes decisores, se puede también ver que los procesos y resultados de evaluación tienen el potencial de dirigirse a un número de necesidades diferentes.

En ese sentido Louis D'hainaut (1985), presentó una taxonomía cognitiva (tabla 1) que puede resultar de gran utilidad para los procesos de planificación y desarrollo curricular y, en consecuencia, para una evaluación orientada al desarrollo de las capacidades, desde las más básicas hasta las de mayor complejidad.

La evaluación en la educación, como todo proceso, ha evolucionado y con el tiempo han aparecido nuevas técnicas e instrumentos que pueden ser usados dentro del contexto de la enseñanza y aprendizaje para, fundamentalmente, recoger evidencias acerca de cómo los estudiantes procesan, complementan tareas y desarrollan capacidades sobre un tema particular (López, Blanca e Hinojosa, 2000). A diferencia de la evaluación tradicional, la nueva tendencia para evaluar permite:
- Enfocarse en documentar el crecimiento del individuo en cierto período de tiempo, en lugar de comparar a los estudiantes entre sí.

- Enfatizar las fortalezas de los estudiantes en lugar de las debilidades.

- Considerar los estilos de aprendizaje, las capacidades lingüísticas, las experiencias culturales y educativas, y los niveles de estudio.

TABLA 1: TAXONOMÍA COGNITIVA DE LOUIS D’HAINAUT

\begin{tabular}{ll}
\hline CAPACIDAD & HABILIDADES \\
\hline 1. Reproducción & $\begin{array}{l}\text { Reproduce, cuenta, expresa, indica, nombra, } \\
\text { registra, cita, describe, relata, enumera, defina, } \\
\text { anota, lee, señala, enuncia, formula. }\end{array}$ \\
\hline 2. Conceptualización & $\begin{array}{l}\text { Localiza, define, relata, resume, clasifica, } \\
\text { compara, diferencia, describe, asocia, completa, } \\
\text { informa, ubica, explica, selecciona, ejemplifica, } \\
\text { discute, indica. }\end{array}$ \\
\hline 3. Aplicación & $\begin{array}{l}\text { Agrupa, emplea, organiza, calcula transforma, } \\
\text { relaciona, desarrolla, experimenta, grafica, } \\
\text { resuelve, usa, diagrama. }\end{array}$ \\
\hline 4. Exploración & $\begin{array}{l}\text { Diferencia, descompone, relaciona, selecciona, } \\
\text { explica, separa, calcula, investiga, indaga, } \\
\text { planifica, revisa. }\end{array}$ \\
\hline 5. Movilización & $\begin{array}{l}\text { Construye, integra, resume, compone, propone, } \\
\text { combina, desarrolla, organiza, formula, crea, } \\
\text { diseña, produce, inventa. }\end{array}$ \\
\hline 6. Resolución de & $\begin{array}{l}\text { Critica, sustenta, determina, categoriza, } \\
\text { interpreta, resuelve, califica. }\end{array}$ \\
\hline Problemas & \begin{tabular}{l} 
ing \\
\hline
\end{tabular}
\end{tabular}

Fuente: Rimari, W. (2009). Las Rúbricas, un innovador y eficiente instrumento de evaluación.

Muchos críticos sostienen que los exámenes tradicionales de respuesta fija no dan una visión clara y veraz sobre lo que los estudiantes conocen; solo permiten evaluar la memoria, la compresión o interpretación del conocimiento, pero no demuestran la habilidad del uso del conocimiento. Además, argumentan que los exámenes estandarizados de respuesta fija ignoran la importancia del conocimiento holístico y la integración del conocimiento, y no permiten evaluar la competencia y las capacidades del educando en objetivos educacionales de alto nivel de pensamiento o de lo que espera la sociedad. Con frecuencia el resultado de las evaluaciones se emplea solo para adjudicar una calificación a los participantes y no entra en las estrategias de enseñanza y de aprendizaje para mejorar los esfuerzos. Para los autores de este trabajo esta es una de las principales razones para la consideración de los sistemas de rúbricas en la evaluación del aprendizaje. 
LAS RÚBRICAS COMO HERRAMIENTA DE VALORACIÓN EN LA EVALUACIÓN DEL APRENDIZAJE

La sociedad en el comienzo del tercer milenio está marcada como la sociedad del conocimiento, que proyecta la formación de sociedades inteligentes que piensan crítica y creativamente, para las cuales es necesario diseñar modelos educativos que propicien el desarrollo del talento, de las inteligencias y del pensamiento innovador (Casas, 2007). Estos modelos requieren también de nuevas propuestas de evaluación. El reto consiste en desarrollar estrategias y técnicas alternativas de evaluación del aprendizaje que respondan a las nuevas demandas.

Entre estas estrategias se encuentran los portafolios, las pruebas situacionales, y los sistemas de rúbricas, siendo estos últimos, en opinión de los autores, una herramienta de gran potencialidad y eficacia para alcanzar los objetivos de aprendizaje en los estudiantes. A continuación se detallan algunos conceptos, notablemente ilustrativos, sobre sistemas de rúbricas.

Según Alan D. Rowe, las rúbricas son una vinculación vital entre la evaluación y el proceso de enseñanza-aprendizaje. Estas operacionalizan la calidad, de manera que se pueda enseñar y guiar a los alumnos con mayor efectividad (Rimari, 2009).

Heidi Goodrich (2000) conceptualiza las rúbricas como una herramienta de evaluación que identifica ciertos criterios para un trabajo. Generalmente, las rúbricas especifican el nivel de desarrollo esperado para obtener diferentes niveles de calidad. Estos pueden estar expresados en términos de una escala (excelente, bueno, necesita mejorar, reprobado) o en términos numéricos $(4,3,2,1)$, que al final se suman para determinar un resultado al que se le asigna una nota (A, B, C, D), o usando la escala vigesimal, es decir 0 a 20); o cualquier otra escala de conveniencia.

Las rúbricas son herramientas de valoración usadas para reflejar el grado de cumplimiento de una actividad o trabajo (Barberá \& De Martin, 2009), en su forma más sencilla se presenta como una tabla de doble entrada que permite unir o relacionar criterios de evaluación, niveles de logro y descriptores. Se tienen columnas y filas, las columnas indican el grado de la calidad y enumera una serie de ítems o áreas que se deben evaluar. Las filas indican los niveles de dominio, en las intersecciones se incluyen descripciones textuales de las cualidades de los resultados y productos en esa dimensión y nivel. Existen otras formas de estructurar las rúbricas, pero funcionan de igual manera. La anterior definición de Barberá y De Martin es asumida por los autores de este trabajo.

Las rúbricas son la mejor herramienta para los procesos de evaluación continua (Blanco, 2008), estas pueden tener varias utilidades, como por ejemplo, clarificar los objetivos del aprendizaje y evaluación; mantenerlos vinculados con los contenidos y las actividades de la asignatura; comunicar a los estudiantes los resultados de aprendizaje esperados y clarificar las expectativas; proporcionarles información clara y específica sobre el trabajo realizado, identificando los logros y aspectos a mejorar; y, cuando son utilizadas por los mismos alumnos, fomentar el desarrollo de competencias meta-cognitivas como la autorregulación del aprendizaje de los alumnos.

Las rúbricas no solo ayudan a realizar evaluaciones cuantitativas sino cualitativas asociadas a criterios preestablecidos, para medir acciones de los evaluados sobre aspectos de una tarea o actividad (Torres y Perera, 2010).

Adicionalmente a lo anterior, las rúbricas pueden ser uno de los mejores mecanismos para realizar autoevaluaciones de mejor calidad (Stevens \& Levi, 2005), con un carácter más cualitativo y procesal. Este tipo de evaluaciones no solo favorecen a una evaluación más sistematizada por parte de los docentes, sino que son una herramienta de extraordinario valor para el desarrollo de competencias de monitorización, autoevaluación y evaluación entre pares, contribuyendo a un mayor entendimiento del propio proceso de aprendizaje y, en definitiva, a una mayor autonomía y autorregulación del estudiante.

El uso de las rúbricas se concibió como una estrategia de innovación didáctica en el proceso de Convergencia Europea (Martínez y Raposo, 2009) de forma que con ellas se pudieran responder tanto a una serie de objetivos vinculados con la formación del alumnado, como a otros más directamente relacionados con la práctica profesional en un momento de investigaciónacción, planteando como primordial:

- Fomentar la implicación de los estudiantes en sus procesos de formación y evaluación.

- Facilitar a los estudiantes criterios que permitan identificar las cosas bien hechas junto con los logros alcanzados. 
- Orientar el trabajo en equipo y dotar de sentido a las tutorías grupales y/o personalizadas.

La inclusión de estos tres elementos en la concepción del uso de rúbricas tiene para los autores de este trabajo un valor notable, y serán considerados como relevantes.

\section{¿POR QUÉ USAR RÚBRICAS?}

El uso de las rúbricas está asociado, fundamentalmente, a cinco ideas:

1. Tiempo corto de respuesta para la retroalimentación.

2. Preparación de los estudiantes para el uso detallado de la retroalimentación.

3. Fomentar el pensamiento crítico.

4. Facilitar la comunicación con otros.

5. Ayudar a perfeccionar las técnicas de enseñanza.

A continuación, y por su centralidad en la fundamentación del uso de rúbricas, se detallan cada una de estas cinco ideas.

TIEMPO DE RESPUESTA PARA LA RETROALIMENTACIÓN

El tiempo de respuesta, sobre las calificaciones de exámenes o trabajos suele ser un punto problemático entre profesores y estudiantes. Los profesores tratan de realizar una calificación personalizada y justa por cada estudiante, a su vez los estudiantes se quejan de que los trabajos no fueron devueltos en un tiempo correcto. Esta constante queja muchas veces presenta a los estudiantes como si su único interés fuera el de obtener su nota final aprobatoria. Según el estudio de Rucker \& Thompson (2003), más allá del interés propio de los estudiantes en su nota final, el tiempo de respuesta corto por parte de los profesores en la calificación de los trabajos es un factor muy importante para producir en los estudiantes cambios positivos en sus trabajos posteriores. Taras (2003) en un trabajo de investigación similar con estudiantes ingleses da cuenta de que una respuesta más rápida eleva los niveles de aprendizaje y desarrolla hábitos personales de auto-evaluación. Por otra parte cuanto mayor sea el retraso en la recepción de información, menor será el efecto de retroalimentación sobre el desempeño (Ilgen, Peterson, Martin, \& Boeschen's, 1981). En los últimos años, otras investigaciones han validado que la retroalimentación oportuna, en lo que respecta al tiempo, facilita el aprendizaje (Black \& Wiliam, 1998).
Sin embargo, el problema radica en la existencia de pocas herramientas que permitan a los profesores ocupar un mínimo de tiempo para dar respuestas a sus alumnos, por ejemplo, un profesor que tenga que evaluar treinta artículos de investigación de treinta estudiantes en un lapso de cuarenta y ocho horas, para que luego cada estudiante pueda analizar y realizar una corrección de sus respectivos trabajos. Para casos como el ejemplo anterior se invoca a las rúbricas, que son herramientas que permiten ahorrar tiempo. Probablemente el ahorro de tiempo sea la primera y principal virtud que se encuentre en el uso de rúbricas, aunque no la única. Al momento de evaluar con rúbricas simplemente se incorporan opciones en cada una de las dimensiones de la rúbrica; luego al momento de calificar el trabajo solo se selecciona la opción más cercana a lo que este refleje de la dimensión que se está analizando. De ser necesario se puede añadir un breve comentario si es que las escalas no cubren con suficiente precisión lo calificado. El proceso de calificación, así como el de retroalimentación de los alumnos sigue siendo el mismo pero el tiempo de respuesta para esto puede ser minimizado hasta en un $50 \%$.

PREPARACIÓN A LOS ESTUDIANTES PARA EL USO DETALLADO DE RETROALIMENTACIÓN

Los estudiantes a menudo solicitan que en sus trabajos calificados se incluya la mayor cantidad de detalles del cómo ha sido calificado, esto le sirve para seguir haciendo lo que están haciendo bien y corregir lo que están haciendo mal. Sin embargo parecería que la mayoría no puede asimilar todo aquello que ocupó gran parte del tiempo del profesor en calificar y a su vez, esto provoca en los profesores un desánimo para incluir más detalles en las evaluaciones, limitándose a emitir declaraciones tales como: carece de cohesión, necesita más referencias, trabajo poco organizado, etc. La retroalimentación es mucho más efectiva en la medida del grado de detalle que se expresa en una evaluación, a diferencia de una calificación a secas (Brinko, 1993). El mismo estudio revela que los resultados de las evaluaciones son muy superiores si se incluye una descripción detallada de los objetivos que se esperan evaluar para obtener una mayor nota o calificación.

Utilizando varias rúbricas de las tareas terminadas, los estudiantes pueden sacar sus propias conclusiones sobre las debilidades en su trabajo y también establecer sus propios 
planes de mejora. Esta es la mejor forma en que se producen el desarrollo de la motivación, y el aprendizaje (Huba \& Freed, 2000).

FOMENTAR EL PENSAMIENTO CRÍTICO

Debido al formato que presentan las rúbricas, los estudiantes pueden observar por sí mismos los patrones de los problemas recurrentes o la mejora continua en sus trabajos, y este autodescubrimiento es uno de los factores de satisfacción más importantes para el uso de las rúbricas y pueden inspirar el patrón de "autoevaluación y mejora de sí mismo", que es el tipo de estudiante que en términos generales se busca tener en clase.

El uso de esta herramienta, en conjunto con un buen asesoramiento académico, puede desempeñar un papel importante para contribuir al desarrollo de los estudiantes de una forma más académica de pensamiento crítico, que es la capacidad de pensar, razonar y hacer juicios de valor sobre la base de una acumulación independiente, precisa de los datos y el enfoque de mente abierta a cada nuevo tema (Huba \& Freed, 2000).

FACILITAR LA COMUNICACIÓN CON OTROS

En general las rúbricas facilitan la comunicación no solamente con los alumnos, sino también con otros profesores y otros grupos de trabajo que intervienen junto con el profesor (otros profesores, ayudantes de cátedra, colaboradores, etc.). Cuando varios profesores tienen que evaluar el mismo contenido a distintos alumnos (por ejemplo profesores de la misma asignatura en distintas secciones del mismo curso) o a los mismos alumnos (por ejemplo, los miembros de un tribunal), el utilizar la misma rúbrica facilita el acuerdo entre profesores, o se localizan mejor las discrepancias si las hay, o simplemente se pueden transmitir cuáles son los ítems que se quieren evaluar realmente.

AYUDAR A PERFECCIONAR LAS TÉCNICAS DE ENSEÑANZA En todo proceso de enseñanza siempre los profesores se hacen las siguientes preguntas: ¿como saber que somos buenos profesores?, ¿cómo podemos ser mejores profesores que otros?, algunas formas estandarizadas ya existentes son una buena vía para obtener las respuestas, sin embargo en muchos casos estas preguntas son realizadas para otros fines diferentes al netamente académico, como por ejemplo una promoción o simplemente mantener la permanencia en la cátedra. En el mejor de los casos estas evaluaciones solo reflejan información sobre si el profesor sabía su materia, o si la clase mantenía la atención del caso o si enviaba el suficiente material de lectura (Huba \& Freed, 2000). En realidad estas encuestas rara vez proveen evidencia o respuestas significativas para contribuir con información acerca de si los estudiantes en realidad están aprendiendo o no en clase. Mediante el uso correcto de rúbricas individuales de cada estudiante, se puede observar su evolución o desarrollo en el tiempo lo cual es en extremo importante, puesto que permite a los profesores encontrar los puntos ciegos u omisiones en una clase, por ejemplo: si la mayoría de los estudiantes de varias clases están dando resultados débiles en el uso de citas o referencias, esto debería ser una llamada de atención para enseñar u obligar al uso de citas o referencias, si usamos estas rúbricas en un lapso de tiempo determinado podremos ver si la estrategia para corregir ese problema específico está dando resultado, se debe cambiar, o mejorar.

CONCLUSIONES

El empleo de rúbricas como herramientas de apoyo a la evaluación del aprendizaje permite un control pormenorizado de los objetivos de aprendizaje, contribuye al desarrollo de técnicas de evaluación a partir de concepciones democráticas.

Las rúbricas permiten lograr mayor celeridad en los procesos de comunicación de resultados de la evaluación del aprendizaje y por tanto facilitan que los estudiantes dispongan de retroalimentación oportuna y personalizada.

\section{REFERENCIAS BIBLIOGRÁFICAS}

Álava, J.; Baquerizo, H.; Hagó, F. (1976). Evolución Histórica de la Vivienda Dentro del Marco del Desarrollo Urbano en la Región de Guayaquil. Guayaquil: Universidad Católica de Santiago de Guayaquil.

Ander-Egg, E. (1993). La planificación educativa. Conceptos, métodos, estrategias y técnicas para educadores. Argentina: Editorial Magisterio.

Barberá, E. y De Martín, E. (2009). Portafolio electrónico: aprender a evaluar el aprendizaje.Barcelona: Editorial UOC.

Black, P. J. \& Wiliam, D. (1998). Assessment and classroom learning. Assessment in Education: Principles, Policy, and Practice, 5(1).

Blanco, A. (2008). Las rúbricas: un instrumento útil para la evaluación de competencias. En Prieto, L (coord.), La enseñanza universitaria centrada en el aprendizaje: estrategias útiles para el profesorado por (171 
188). Barcelona: Octaedro-ICE de la Universidad de Barcelona.

Blanco, F. (1994). La evaluación en la educación secundaria. Salamanca: Amasú Ediciones.

Brinko, K.T. (1993).The Practice of Giving Feedback to Improve Teaching: What Is Effective? Recuperado de http://www.jstor.org/stable/2959994.

Casas, Luis D. (2007). Evaluación de capacidades y valores en la sociedad del conocimiento. Lima: Editorial Norma.

Coffman, W. E. (1971). Essay examinations. En Thorndike, R.L (Ed.) Educational Measurement. Washington, DC: American Council on Education.

D'Hainaut, L. (1985). Objetivos didácticos y programación. Barcelona: Oikos Tau.

Dubois, P. H. (1970). A History of Psychological Testing. Boston: Allyn Bacon.

Escudero, T. (2003). Desde los test hasta la investigación evaluativa actual. Un siglo, el XX, de intenso desarrollo de la evaluación en educación. Revista electrónica de investigación y evaluación educativa, 9(1). Recuperado de www.uv.es/RELIEVE/v9n1/RELIEVEv9n1_htm.

González, M. (2002). La evaluación del aprendizaje. Recurso Educativo de la Maestría en Educación Superior. CEPES/Universidad de La Habana.

Goodrich, Heidi (2000). "Using Rubrics to Promote Thinking and Learning" artículo en línea. Recuperado de http://www.ascd.org/publications/educational_ leadership/feb00/vol57/num05/Using_Rubrics_to_ Promote_Thinking_and_Learning.aspx.

Gvirtz, S y Palamidessi, M. (2006). El ABC de la tarea docente: curriculum y enseñanza. (Tercera edición). Buenos Aires: AIQUE Grupo Editor S.A.

Huba, M. E. \& Freed, J. E. (2000). Learner-Centered Assessment on College Campuses - Shifting the Focus from Teaching to Learning. Boston: Allyn and Bacon.

Ilgen, D. R., \& Moore, C. F. (1987). Types and choices of performance feedback. Journal of Applied Psychology, 72, 401-406.
Ilgen, D. R., Peterson, R. B., Martin, B. A., \& Boeschen, D. A. (1981). Supervisor and subordinate reactions to performance appraisal sessions. Organizational Behavior and Human Performance, 28, 311-330.

Litwin, Edith. (2004). Las configuraciones didácticas. Una nueva agenda para la enseñanza superior. Buenos Aires: Paidós.

Martínez, M.E. y Raposo, M. (diciembre,2009).La rúbrica en la tutorización de trabajos en grupo: opiniones y valoraciones. En IV Jornadas de Innovación Didáctica, Universidad de Vigo, España

Osorio, B., Muñoz, J., Álvarez, F., y Arévalo, C. (2005). Metodología para elaborar objetos de aprendizaje e integrarlos a un sistema de gestión de aprendizaje. Recuperadodehttp://sedici.unlp.edu.ar/bitstream/ handle/10915/38644/Documento_completo. pdf?sequence $=1$

Rimari, W. (2009). Las Rúbricas, un innovador y eficiente instrumento de evaluación. Revista Pedagógica "San Jerónimo". Recuperado de https://es.scribd. com/doc/16918521/LA-RUBRICA-instrumento-deevaluacion-Wilfredo-Rimari

Rucker, M.L., Thompson, S. (2003). Assessing student learning outcomes: an investigation of the relationship among feedback measures. College Student Journal, 37, 400-405.

Santos, M. A. (1999). 20 paradojas de la evaluación en la universidad española. Recuperado de http://www. uva.es/ aufop/aufopweb.htm.

Stenhouse, L. (1984). Investigación y desarrollo del curriculum. Madrid: Morata.

Steven, D. \& Levi, A. (2005) Introducction to Rubrics, Sterling, VA

Sunberg, N. D. (1977). Assessment of person. NJ: Prentice Hall.

Taras, M. (2003). To feedback or not to feedback in student self-assessment. Assessment and Evaluation in Higher Education, 28(5), 549-565.

Torres, J. J. y Perera, V. H. (2010). La rúbrica como instrumento pedagógico para la tutorización y evaluación de los aprendizajes en el foro online en educación superior. Píxel-Bit. Revista de Medios y Educación, 36, 141149. 